\title{
Measuring the Blood Sugar Level and Time (BSLT) Using Mobile Strategy to Remote Monitoring of DIABETES Chronic Diseases for Children in North African Countries
}

\author{
${ }^{1}$ Osama E. Sheta, ${ }^{2}$ Majzoob Kamal Aldein Omer and ${ }^{3}$ Mohamed Sir Elkhtem \\ ${ }^{1}$ Dept. of Mathematics (Computer Science) Faculty of Science, Zagazig University, Zagazig, Egypt; \\ ${ }^{2}$ Dept. of Information Technology, AL Neelain University, Khartoum, Sudan; \\ ${ }^{3}$ Dept. of Information Technology, National Ribat University, Khartoum, Sudan \\ oesheta75@gmail.com; dr.majzoob_kamal@neelain.bu.sd; dr.mohsir@gmail.com
}

\begin{abstract}
Chronic diseases for children such as DIABETES is a challenge for parents in all third world countries. Especially that percentage of giving birth to children with chronic diseases is increasing dramatically in North African countries. Thus the official task of parents to help their children in how to live and deal the condition with this chronic disease. In this paper we propose a child DIABETES monitoring system which is integrated sensors and smart phone to facilitate the management of chronic disease - DIABETES. The system automatically collects physical signs, such as Blood Glucose level. It allows users, especially children with diabetes to conveniently record daily test results and track long term health condition changes regardless of their locations. All the procedures are performed automatically without entering the user (Patient) any data on the system manually. Of the most important objectives of the application is help the parents to monitoring the level of sugar in the blood for their child at any time and alert them automatically to any risk.
\end{abstract}

Keywords: Mobile Strategy, Wearable Sensors, Chronic Disease, DIABETES, Mobile-Health, Blood Sugar Level and Time (BSLT).

\section{Introduction}

Chronic diseases are becoming the heaviest burden to healthcare systems worldwide. World Health Organization [1, 2] reported that, from 57 million global deaths in 2008, 63 percent were due to chronic diseases mainly caused by pulmonary diseases, heart failure, cancer, and diabetes. In the next several decades major chronic diseases will continue to increase the morbidity and mortality rate in the entire world [3]. Obviously, traditional model of episodic care in clinic and hospital-based settings is suboptimal for improving chronic disease outcomes [4]. Indeed, in daily life, the prevention and treatment of chronic diseases take place outside of traditional clinical settings. Therefore, more appropriate and higher quality nursing interventions are required to support self-care, to reduce chronically ill hospitalization, and to eventually ease the burden of chronic diseases. 
Diabetes is a group of metabolic diseases which are characterized as elevated levels of blood glucose (hyperglycemia) caused by defects in insulin secretion, insulin action, or both [5]. Complications of diabetes are severe such as retinopathy, nephropathy, and neuropathy. For this reason main goal of diabetes care is to achieve normal blood glucose levels to reduce the development of undesired complications, thus maintaining good quality of life. In order to achieve this goal diabetes' inventions have been categorized as nutritional management, exercise, monitoring, pharmacological therapy and education [5].

Type 1 diabetes mellitus (T1DM) is one of the most common endocrine and metabolic conditions in childhood. Data from large epidemiological studies worldwide indicate that on an annual basis, the overall increase in the incidence of T1DM is around 3\% and about 78000 children under age 15 years develop T1DM worldwide [6]. Among Eastern Mediterranean and Middle Eastern countries, the largest contribution to the total number of estimated childhood T1DM cases comes from Egypt which accounts for about a quarter of the region's total. The incidence varies between 8/100000 per year (Egypt) in children under the age of 15 years [7].

In recent years, small and wearable sensors have become widely available, which allows monitoring of patients' blood glucose. In addition, contemporary smart phones incorporate unforeseen computational powers which include internal database, voice recognition, GPS position and continuous access to local wireless networks and the Internet. This makes newer cellular phones a very promising mobile platform for advanced applications. This provides advantages in our research: (1) facilitate the exchange of information between children diabetics and their parents; (2) providing easy access to the children diabetics from any place and at any time. Thus, it is possible to bring wearable sensors with Bluetooth or Wi-Fi and smart phone together to create a personalized, integrated and collaborative care system, which would improve children diabetic's long-term health.

In this paper we design and integrated personal-care system, and collaborative self-monitoring and management of diabetes. The system monitors blood sugar levels. Also, this system is based on permanent contact between children diabetics, and their parents, and the various health care providers so that they can now provide collaborative patient care. In section 2, we summarize some related work to wearable sensors and pervasive health monitoring systems. Section 3 describes the integrated architecture design including software, hardware and communication interfaces among different components of the system, and gives the software architecture design in mobile phone. In section 4, we describe the behavioral modification flowchart and the case study. Finally, conclusion and future work are presented in section 5 .

\section{A Review of Literature}

In the last few years a clear improvement in the field of e-health monitoring systems can be seen. Many deferent types of e-health monitoring systems have been devolved and proposed, especially of chronic disease - diabetes. The AlarmNet architecture proposed in [9] [10] is a common architecture which integrates body sensor networks, environment sensors, back-end data management and analysis system, and local or remote user interface. 
Osama E. Sheta, Majzoob Kamal Aldein Omer and Mohamed Sir Elkhtem; Measuring the Blood Sugar Level and Time (BSLT) Using

Mobile Strategy to Remote Monitoring of DIABETES Chronic Diseases for Children in North African Countries. Journal of Biomedical Engineering and Medical Imaging, Volume 2, No 4, August (2015) , pp 1-8

Other systems in [11] [12] [13] [14], the authors proposed a similar architecture as AlarmNet. Body sensor networks are composed of wearable and wireless sensing and monitoring devices. These wearable devices are used to monitor the person's blood glucose level, blood pressure, ECG or to detect fall.

Using mobiles with a multi-access service for the management of diabetic patients was proposed in [15], which was designed to collect data, either manually or automatically from the blood glucose meter; to monitor blood glucose levels; to suggest insulin dose adjustment when needed; to deliver monitoring data to a health care center.

In [16] [17] [18] [19] [20], they proposed similar architectures for monitoring heart disease. In those systems, the cell phone acts as the controller and gateway. The cell phone collects data from wearable biosensors or independent meters like glucometer and insulin pump through Bluetooth. Also, the cell phone could access Internet delivering collected data to a specific data center through Wi-Fi wireless network or CDMA/3G mobile network.

\section{Problem Description}

Digital services and mobile applications that are being developed in this paper will help parents to control their children diabetes, especially if the patient is outside of the home, for example in the school. The first type of treatment of this disease is not giving insulin only, but by teaching and education, which would help from a physician for success in the treatment of the child or the child infected with diabetes. Therefore, continuous self-monitoring of the Blood Glucose (Blood Sugar), daily diet, physical activity and medicine intake are crucial for the management of diabetes.

For the young child or teen who does not want to write values (Patterns of high or low Blood Sugars) as show in figure 1, it is often acceptable for the parents to bringing the results to clinic, which allow team work together most effectively to achieve good diabetes management.

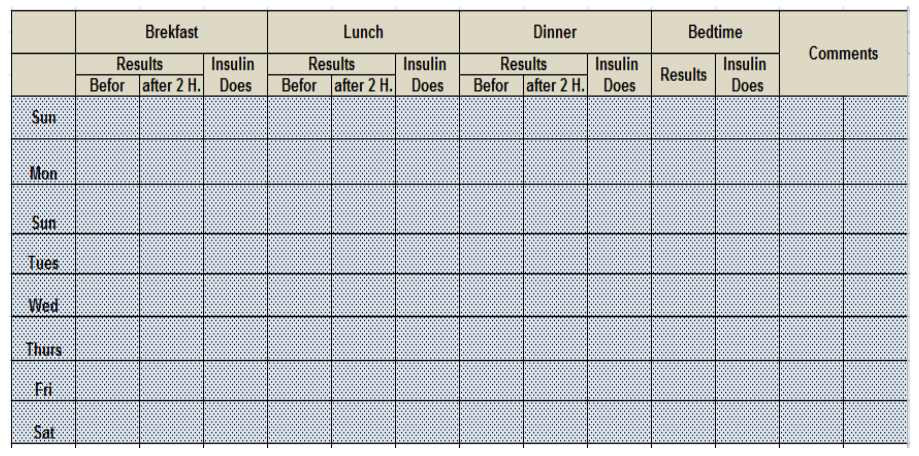

Figure 1: Daily Record Sheet

American Diabetes Association suggested Blood sugar levels of the children diabetics as shown in Table 1. 
Table 1: suggested Blood sugar levels

\begin{tabular}{|c||c|c||c|c||}
\hline \multicolumn{1}{|c||}{} & \multicolumn{2}{c|}{$\begin{array}{c}\text { Fasting (a.m.) or } \\
\text { no food for } \mathbf{2} \text { hours }\end{array}$} & $\begin{array}{c}\text { Bedtime (before bedtime } \\
\text { snack } \\
\text { or during the night) }\end{array}$ \\
\hline \hline Age (years) & $\mathbf{M g} / \mathbf{d l}$ & $\mathbf{M m o l} / \mathbf{l}$ & $\mathbf{M g} / \mathbf{d l}$ & Mmol/I \\
\hline \hline Below 5 & $80-200$ & $4.5-11.1$ & Above 150 & Above 8.3 \\
\hline \hline $5-11$ & $70-180$ & $3.9-10.0$ & Above 130 & Above 7.3 \\
\hline $\begin{array}{c}12 \text { and } \\
\text { above }\end{array}$ & $70-150$ & $3.9-8.3$ & Above 130 & Above 7.3 \\
\hline
\end{tabular}

\section{System Model and Design:}

In this section, we introduce system architecture and the software design of each component including the sensors used in the system and communication technologies among different components. Figure 2 shows the overall picture of the system architecture of our proposed mobile personal health care system for children with diabetes.

The system architecture includes four components: wearable sensors, 3/4G smart phone, web server and on-line data storage - daily record sheet. For example, when the Sensor takes a blood glucose test, then it will transfer the test result to the smart phone. It's will store this test result into the internal database and it will send the test result through the web service. Finally, the parents will get a notification to look at the test result and give the immediate feedback to the children.

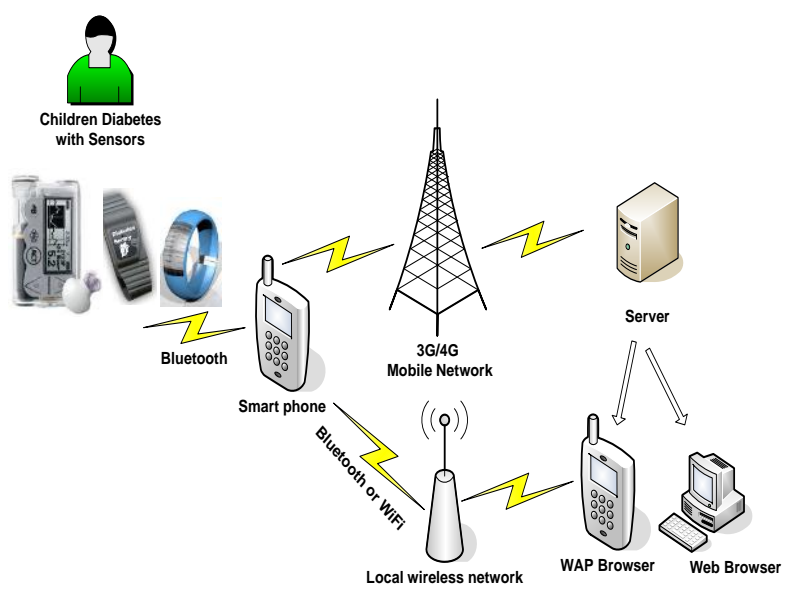

Figure 2: System Architecture of Mobile Personal Health system

\subsection{Wearable sensor}

Wearable sensors allow convenient, continuous and non-intrusive monitoring of people with diabetes for their test results, medicines, daily diet and exercise. There are many sensors like, MyGlucoHealth ${ }^{\circledR}$ Meter [21] to trace patients' blood glucose level.

\subsection{G smart phone}

4/3G smart phone will gather, store and display statistical data from wearable sensors, update daily test results, and get feedback and notification from caregivers. There are two ways for smart phone to interact 
Osama E. Sheta, Majzoob Kamal Aldein Omer and Mohamed Sir Elkhtem; Measuring the Blood Sugar Level and Time (BSLT) Using

Mobile Strategy to Remote Monitoring of DIABETES Chronic Diseases for Children in North African Countries. Journal of Biomedical Engineering and Medical Imaging, Volume 2, No 4, August (2015) , pp 1-8

with daily record sheet. In local area, smart phone detects existing wireless Access Point (AP) and accesses Internet via Wi-Fi wireless networks. Otherwise it resorts to 4G mobile network.

\subsection{Daily record sheet}

Daily record sheet for patients to maintain their personal data like test results. It also allows appropriate access as needed with the permission of the patient to different health care providers. In this system, it is used to manage Blood sugar levels and facilitates information sharing between patients and multiple providers.

\subsection{Software Strategy:}

The new Strategy allows access to healthcare systems online including scheduling, and tele health monitoring from the home environment. Typically this is done via an easy-to-access Patient behavior as shown in Figure 3. The Following steps illustrate System Procedures to monitoring and controlling the patient's condition.

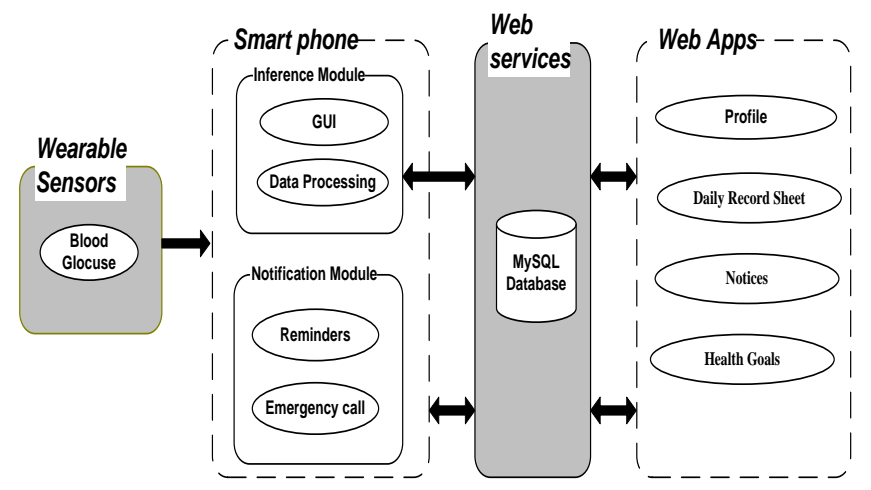

Figure 3: Software Framework of Mobile Children Diabetes System

The first Processing: is to read Blood Sugar Level and Time (BSLT), There are very important conditions for a reliable system to give useful results for patient monitoring such as, if the age of the child was less than 5 years and then is being read blood sugar at bedtime and the rate over 200, The system alerts the parents of the seriousness of the case and determines child's insulin dosage, otherwise if the Sugar rate less than 150, this case are considered less serious and the system alert the parents to gives him the Snack.

On the other hand, if the child did not eat or the System read the Blood Sugar Level and Time indicators two hours after eating, and the rate over 200 , then the system alerts the parents of the seriousness of the case and determines child's insulin dosage, otherwise if the rete less than 80 , the system alert the parents to gives the child Snack.

The Second Processing: if the age of the child over than 5 years and then is being read blood sugar at bedtime and the rate over 180, The system alerts the parents of the seriousness of the case and determines child's insulin dosage, otherwise if the Sugar rate less than 130, this case are considered less serious and the system alert the parents to gives him the Snack.

Also if the child did not eat or the System read the Blood Sugar Level and Time indicators two hours after eating, and the rate over 180, then the system alerts the parents of the seriousness of the case and 
determines child's insulin dosage, otherwise if the rate less than 70 then the system alert the parents to gives the child Snack. The previous steps shown in figure 4.

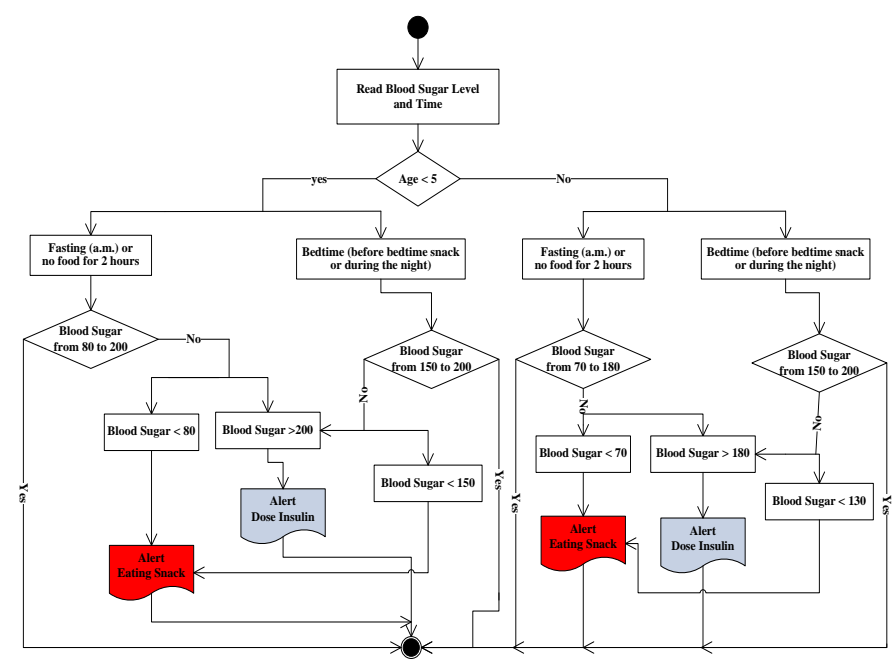

Figure 4: New Strategy to Monitoring and Controlling the BSLT

\section{Conclusion}

In this paper, we proposed an enhancement model to increase the efficiency of the Mobile strategy for DIABETES Chronic diseases for children in North African countries, and to increase the efficiency of the operations in mobile health, our results from this model to create interactive and dynamic application and how to build Mobile Application that is ability to track, monitoring and controlling DIABETES Chronic diseases for children using Blood Sugar Level and Time (BSLT) easy to use by the parents, also we propose a new strategy to develop Mobile Application by using the Wearable sensor and Daily record sheet, WEB server, WAB Server and on-line data storage applied by smartphone platform to monitoring and controlling the level of sugar in the blood for the child at any time and alert the user (parents) automatically to any risk, this services delivery systems and opening up services to provision of new services, such are remote monitoring and tele health. Finally, the using of proposed model does not just support by mobile service, it can be integrated with WEB and WAB services.

\section{REFERENCES}

[1] WHO. 2011. mHealth New horizons for health through mobile technoligies.

[2] WHO. Chronic diseases. Referred 13.07.2012. http://www.who.int/topics/chronic_diseases/en/.

[3] Global status report on noncommunicable diseases2014. http://www.who.int/nmh/publications/ncd_report2014/en/index.html.

[4] Estrin, D. \& Sim, I. 2010. Open mHealth Architecture: An Engine for Health Care Innovation. SCIENCE. Published by AAAS. 
Osama E. Sheta, Majzoob Kamal Aldein Omer and Mohamed Sir Elkhtem; Measuring the Blood Sugar Level and Time (BSLT) Using

Mobile Strategy to Remote Monitoring of DIABETES Chronic Diseases for Children in North African Countries. Journal of Biomedical Engineering and Medical Imaging, Volume 2, No 4, August (2015) , pp 1-8

[5] Maschak-Carey, B. J. 2003. Medicl-Surgical Nursing. 10th edition. Chapter 41: Assessment and Management of Patients with Diabetes Mellitus. ISBN-10: 0781731933.

[6] International Diabetes Federation. The IDF Diabetes Atlas. 5th ed. http://www.idf.org/idfdiabetes-atlas-fifth-edition. Date of last access 23th April 2013. Brussels: International Diabetes Federation; 2011.

[7] Soltèsz G, Patterson C, Dahlquist G. Diabetes Atlas, 3rd ed. Brussels: International Diabetes Federation; 2006. Global trends in childhood type 1 diabetes; pp. 153-190.

[8] El-Zanaty F, Way A. Demographic and Health Survey 2008. Calverton, Maryland: Egypt, Ministry of Health, EL-Zanaty and Associates and Macro International 2009; 2009.

[9] Anthony D. Wood, John A. Stankovic, Gilles Virone, Leo Selavo, Zhimin He, Qiuhua Cao, Thao Doan, Yafeng Wu, Lei Fang, and Radu Stoleru, Context-Aware Wireless Sensor Networks for Assisted Living and Residential Monitoring, IEEE Network, issue 4, pp 26-33, July-August 2008.

[10] G. Virone, A. Wood, L. Selavo, Q. Cao, L. Fang, T. Doan, Z. He, R. Stoleru, S. Lin, and J.A. Stankovic, An Advanced Wireless Sensor Network for Health Monitoring, Distributed Diagnosis and Home Healthcare, 2006. D2H2. 1st Transdisciplinary, pp 2-5, Conference on 2-4 April 2006.

[11] Hsu-Yang Kung, Chi-Yu Hsub and Mei-Hsien Lin, Sensor-based Pervasive Healthcare System: Design and implementation, Journal of High Speed Networks 16, pp 35-49 35 IOS Press, 2007.

[12] Shanshan Jiang, Yanchuan Cao, Sameer Iyengar, Philip Kuryloski, Roozbeh Jafari Yuan Xue, Ruzena Bajcsy, Stephen Wicker, CareNet: An Integrated Wireless Sensor Networking Environment for Remote Healthcare, TRUST Autumn 2007, pp 9:1-9:3 Conference on October 10-11, 2007.

[13] Tia Gao, Dan Greenspan, Matt Welsh, Radford R. Juang and Alex Alm, Vital Signs Monitoring and Patient Tracking Over a Wireless Network, In Proceedings of the 27th Annual International Conference of the IEEE EMBS, pp 102-105, Conference on September 2005.

[14] Subhas C. Mukhopadhyay*, Anuroop Gaddam and Gourab S. Gupta, Wireless Sensors for Home Monitoring - A Review, 2008 Bentham Science Publishers Ltd and Recent Patents on Electrical Engineering, pp 32-39, 2008.

[15] Going Mobile with a Multiaccess Service for the Management of Diabetic Patients, Giordano Lanzola, Davide Capozzi, Giuseppe D’Annunzio, Pietro Ferrari, Riccardo Bellazzi and Cristiana Larizza, Journal of Diabetes Science and Technology, Volume 1, Issue 5, September 2007. 
[16] Valérie Gay, Peter Leijdekkers, Edward Barin, A Mobile Rehabilitation Application for the Remote Monitoring of Cardiac Patients after a Heart Attack or a Coronary Bypass Surgery (PETRA'09), Article No. 21, Corfu, Greece, June 09-13, 2009.

[17] Wan-Young Chung, Chiew-Lian Yau, Kwang-Sig Shin, and ksto Myllyla, A Cell Phone Based Health Monitoring System with Self Analysis Processor using Wireless Sensor Network Technology, Proceedings of the 29th Annual International Conference of the IEEE EMBS Cite Internationale, pp 3705-3708, Lyon, France August 23-26, 2007.

[18] Benny P.L.Lo, Surapa Thiemjarus, Rachel King, Guang-zhong Yang, Body Sensor Network - A Wireless Sensor Platform for Pervasive Healthcare Monitoring, Adjunct Proceedings of the 3rd International conference on Pervasive Computing (PERVASIVE'05), pp. 77-88, May 2005.

[19] Peter Leijdekkers, Valerie Gay and Edward Barin, Trial Results of a Novel Cardiac Rhythm Management System Using Smart Phones and Wireless ECG Sensors. ICOST 2009, LNCS 5597, pp. 32-39, 2009.

[20] W. Chung, et al., A Cell Phone Based Health Monitoring System with Self Analysis Processor using Wireless Sensor Network Technology (29th EMBC), pp. 3705-3708, 2007.

[21] MyGlucoHealth ${ }^{\circledR}$ Meter,http://www.myglucohealth.net/myglucohealth-wireless.html, Accessed October 03, 2010. 